\title{
Robust Routing Using Electrical Flows
}

\author{
Ali Kemal Sinop \\ asinop@google.com \\ Google Research \\ Sreenivas Gollapudi
sgollapu@google.com
Google Research
}

\begin{abstract}
Generating alternative routes in road networks is an application of significant interest for online navigation systems. A high quality set of diverse alternate routes offers two functionalities - a) support multiple (unknown) preferences that the user may have; and b) robust to changes in network conditions. We address the latter in this paper. The main techniques that produce alternative routes in road networks are the penalty and the plateau methods, with the former providing high quality results but being too slow for practical use and the latter being fast but suffering in terms of quality. In this work we propose a novel method to produce alternative routes that is fundamentally different from the aforementioned approaches. Our algorithm borrows concepts from electrical flows and their decompositions. We evaluate our method against the penalty and plateau methods, showing that it is as fast as the plateau method while also recovering much of the headroom towards the quality of the penalty method. The metrics we use to evaluate performance include the stretch (the average cost of the routes), the diversity, and the robustness (the connectivity between the origin and destination) of the induced set of routes.
\end{abstract}

\section{CCS CONCEPTS}

- Theory of computation $\rightarrow$ Shortest paths; • Information systems $\rightarrow$ Geographic information systems.

\section{KEYWORDS}

routing, road networks, algorithms, electrical flows, alternates

\section{ACM Reference Format:}

Ali Kemal Sinop, Lisa Fawcett, Sreenivas Gollapudi, and Kostas Kollias. 2021. Robust Routing Using Electrical Flows. In 29th International Conference on Advances in Geographic Information Systems (SIGSPATIAL '21), November 2-5, 2021, Beijing, China. ACM, New York, NY, USA, 11 pages. https://doi. org $/ 10.1145 / 3474717.3483961$

\section{INTRODUCTION}

In the last two decades, computer based navigation systems, whether it be web-based, hand-held or in-car device, became a critical component of our lives. Computing routes that are resistant to changes

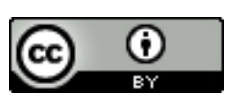

This work is licensed under a Creative Commons Attribution International 4.0 License. SIGSPATIAL '21, November 2-5, 2021, Beijing, China

(C) 2021 Copyright held by the owner/author(s).

ACM ISBN 978-1-4503-8664-7/21/11.

https://doi.org/10.1145/3474717.3483961

\author{
Lisa Fawcett \\ lfawcett@google.com \\ Google Research \\ Kostas Kollias \\ kostaskollias@google.com \\ Google Research
}

in the network is central to any navigation system. In fact, the dynamic shortest path problem is a notoriously hard problem to solve efficiently. In this study, we approach this problem using a set of paths instead of a single "best" path between the source and destination. Therefore, the goal is to compute a set of paths from which a navigation engine is able to always find a path to the destination. We show that this can be achieved by efficiently computing a diverse set of effective alternates between the source and destination. While the efficiency addresses the path query latency, effectiveness addresses the length of the paths produced as well as their robustness to changes in the network.

Unlike shortest paths, there is no single criteria to evaluate the quality of a set of alternates routes. There are three natural properties a good set of alternates should have: 1) Each alternate path should not be too long compared to the shortest path (stretch). 2) The alternates should be sufficiently different from each other (diversity). 3) There should be a good route among the alternates even under changing traffic conditions, such as road closures (robustness).

Finding shortest paths is a well studied problem in computer science. Modern techniques, such as Contraction Hierarchies [13] or Customizable Route Planning [8], can compute the optimal routes in road networks on the order of tens of milliseconds. Such algorithms are based on a preprocessing step, where they exploit the uniqueness property of the shortest path distances and augment the original graph so that the subsequent shortest path queries could be carried out fast.

On the other hand, research on finding alternate routes started rather recently. These work fall under two different approaches. The first approach [2] is based on using via-nodes (think of them as intermediate stops), and computes alternative paths from source to destination that pass through different via-nodes. One popular approach is the plateau method [1], which performs a bi-directional Dijkstra and uses the edges which are present in both trees (plateau edges) as via nodes. The second approach, called penalty methods, is based on finding shortest paths repeatedly, and increasing the weight of edges around these shortest paths [4]. Our method is a fundamentally different approach than the plateau and penalty methods for generating alternates, and it is based on electrical flows.

\subsection{Our Contributions}

In this paper, we propose a new algorithm for generating fast, high quality, diverse and robust set of alternate paths. Our algorithm is based on finding electrical flows, and uses flow decomposition techniques to identify a good set of alternate routes. Furthermore, 
we give efficient preprocessing algorithms so as to speedup the subsequent queries.

In order to evaluate the robustness aspect of alternate paths, we propose two notions of robustness, mimicking the (unexpected) closures in the road networks. The first one is the robustness of the alternate graph, where we measure how many random edges can be removed from the road network before the source and destination becomes disconnected in the alternate graph. The second one is the robustness of the alternate paths. Here we measure the number of alternate paths that survive (in expectation) random edge deletions, for various probabilities.

Finally, we evaluate our algorithm against the plateau and penalty methods in terms of running time, stretch, diversity and robustness.

\subsection{Related Work}

In this section, we will review the related work on alternate generation algorithms and electrical flows.

1.2.1 Alternates. The first approach to alternatives routes was based on using different objective functions, such as best travel-time, minimum distance, road quality, scenery and so on [5]. However this approach is not scalable, as even for a small set of features, there will be exponentially many objective functions. Moreover many of the potential criteria are not independent of each other.

A second approach is the $k$-shortest path problem [27]. This approach has been described as less effective [4] for alternate generation in road networks: Most of the alternates generated will end up being minor modifications of the original path (such as exiting and immediately re-entering a highway) and it will be very unlikely to find a decent alternate in the first few hundred paths.

The penalty method was first introduced by de la Barra and Perez [7], where only the shortest path was penalized. On its own, this could result in a large number of small detours, similar to $k$-shortest paths. Bader et al. [4] modified this method to apply the penalty to the arcs adjoining the shortest paths also. The union of the shortest paths found form the alternative graph. Paraskevopoulos and Zaroliagis [21] suggested modifications to the penalization scheme to obtain higher quality alternative graphs. They also introduced pruning techniques for faster computation of alternates.

The via-node and plateau methods $[1,2,19]$ are the most popular approaches for alternates generation. The goal is to find a single intermediate stop node (via-node) and take the shortest path from source to destination that goes through the via-node. Abraham et al. [2] define a set of criteria the alternative route needs to satisfy to be a viable candidate. One problem with this approach is the identification of good via-nodes. In the plateau method, bi-directional Dijkstra is performed and the common edges are chosen as the via-nodes. This results in transitions that look natural. For a more in-depth description of both plateau and penalty methods, we refer the reader to Kobitzsch's thesis [16].

1.2.2 Electrical Flows. In 2004, Faloutsos et al. [12] used electrical flows for finding connection graphs, which are small subgraphs that best capture the relationship between a pair of nodes.

After the emergence of near-linear time Laplacian solvers with the seminal work of Spielman and Teng [25], and due to their wellknown connections with random walks, electrical flows received a lot of attention from the algorithms community. In the context of routing, electrical flows were successfully used for oblivious routing: In this model of multi-commodity flow, the demands are not known in advance and come in an online, adversarial fashion. The performance of the algorithm is measured with respect to the best possible routing (competitiveness) under various measures of congestion. Harsha et al. [14] showed that under linear congestion, electrical flow routing is $O(\log n)$-competitive. Lawler and Narayanan [18], Kelner and Maymounkov [15] showed that electrical flow routing achieves $O(\log n)$-competitiveness for congestion and other measures based on norms for expander graphs. Schild et al. [23] showed that, for any graph, the average stretch of an edge under electrical flow is $O\left(\log ^{2} n\right)$, and that for grids, the stretch is $O(\log n)$ for any pair.

\section{PRELIMINARIES}

Let $G=(V, E)$ be an undirected, simple graph on $n$ nodes and $m$ edges (which are oriented arbitrarily), with non-negative weights, $w: E \rightarrow \mathbb{R}_{+}$on its edges. We assume the weights represent distances or costs of traversing the corresponding edges. For simplicity, we will assume $G$ is connected. For a given subset of nodes $S \subseteq V$, we will use $G[S]$ to denote the subgraph induced in $G$ by $S$. For a node $s$, we use $N(s)$ to denote its neighbors.

Flows. Since we will be working with distributions over paths, it will be more convenient for us to work with flows. We define a flow on $G, f \in \mathbb{R}^{E}$, as a function on the edges of $G$ such that, on any edge $e=(u, v), f_{e}$ equals the net flow from $u$ towards $v$ (which might be negative if the flow is from $v$ to $u)$. For such an edge $e=(u, v)$, we will use the notation $f_{u \rightarrow v}:=f_{(u, v)}$ and $f_{v \rightarrow u}:=-f_{(u, v)}$. All flows we consider will be circulation-free, which means the sum of flows around any cycle is always 0 . A flow $f$ is a unit flow from $s$ to $t$ if:

- The net flow on $s$ is $1, \sum_{u \in N(s)} f_{s \rightarrow u}=1$.

- The net flow on $t$ is $-1, \sum_{u \in N(t)} f_{s \rightarrow u}=-1$.

- The net flow is 0 on any other node $v, \sum_{u \in N(v)} f_{v \rightarrow u}=0$.

Note that any path $p$ between $s$ and $t$ naturally corresponds to a flow, with the flow value on edge $e=(u, v)$ being +1 if $(u, v) \in p$, -1 if $(v, u) \in p$ and 0 otherwise.

Any circulation-free unit flow $f$ from $s$ to $t$ can be written as a convex combination of paths from $s$ to $t$, in which all paths have the same direction of flow on every edge and $f=\sum_{p \in \mathcal{P}_{s t}} \alpha_{p} f_{p}$, for some non-negative values $\alpha_{p}$ which sum up to 1 . Here $\mathcal{P}_{s t}$ is the set of simple paths from $s$ to $t$ and $f_{p}$ is the flow corresponding to path $p$. Note that the choice of $\alpha$ 's is, in general, not unique; however it is always possible to decompose a unit flow into a convex combination of paths.

Before we begin a more formal treatment of this routing algorithm, we need to introduce several matrices central to the study of electrical networks [26].

Vector and Matrix Notation. We assume the rows and columns of vectors and matrices are associated with sets. We will use $x \in \mathbb{R}^{A}$ to denote a vector whose rows correspond to the set $A$ : For any $B \subseteq A$, we will use $x_{B} \in \mathbb{R}^{B}$ to denote the restriction of $x$ to $B$. Similarly we use $M \in \mathbb{R}^{A \times B}$ to denote a matrix whose rows and columns are associated with the sets $A$ and $B$, respectively. For any $C \subseteq A, D \subseteq B$, we will use $M_{C, D} \in \mathbb{R}^{C \times D}$ to denote the minor of $M$ corresponding 
to rows $C$ and columns $D$. We use $M^{T}, M^{-1}$ and $M^{\dagger}$ to denote the transpose, inverse and (if not invertible) pseudo-inverse of $M$, resp.

Conductance Matrix. We define the conductance matrix, $C \in$ $\mathbb{R}^{E \times E}$, as the diagonal matrix that has the conductance of each edge along its diagonal. In particular, $C_{e, e}=\frac{1}{w_{e}}$ (for example, the conductance of an infinitely long edge is 0 ).

Discrete Gradient and Divergence Operators. We will use $B \in$ $\mathbb{R}^{E \times V}$ to denote the signed edge-node incidence matrix of $G$ : Even though the graph is undirected, we assume the edges are oriented arbitrarily. Each row of $B$ is associated with an edge, $e \in E$; and each column of $B$ is associated with a node, $v \in V$. For any edge $e=(u, v) \in E$ and node $x \in V$, the corresponding entry of $B$ is given as:

$$
B_{e, x}= \begin{cases}+1 & \text { if } x=u, \\ -1 & \text { if } x=v, \\ 0 & \text { else. }\end{cases}
$$

With the help of the incidence and conductance matrices, $B$ and $C$, we can define the discrete gradient operator on $G, \nabla_{G} \in \mathbb{R}^{E \times V}$, as $\nabla_{G}:=C B . \nabla_{G}$ sends functions on $V$ to functions on the edge set, $E$. In particular, for any vector on nodes, $x \in \mathbb{R}^{V}$ and any edge $e=(u, v) \in E,\left(\nabla_{G} x\right)_{e}=\frac{1}{w_{u v}}\left(x_{u}-x_{v}\right)$. Similarly, we can view $B^{T}$ as a discrete divergence operator: Given any flow $f \in \mathbb{R}^{E}, B^{T} f$ measures the net flow on each node. For example, if $f$ is a unit flow from $s$ to $t$, then $B^{T} f=\chi_{s}-\chi_{t}$, where $\chi_{s}$ is the indicator vector for node $s$. We will use $\Delta_{G} \in \mathbb{R}^{V \times E}$ to denote this matrix, $\Delta_{G}=B_{G}{ }^{T}$. When the graph is clear, we will drop the subscripts, and use $\nabla, \Delta$ instead.

Laplacian Matrix. Next, we define the Laplacian matrix $L$ associated with $G$ as $L_{G}=\Delta_{G} \nabla_{G}$. For any vector $x \in \mathbb{R}^{V}$, the quantity $x^{T} L x=\sum_{(u, v) \in E} w_{u v}^{-1}\left(x_{u}-x_{v}\right)^{2}$ measures the energy dissipated by the electrical network corresponding to $G$ if the potential at each node was equal to $x$. From this discussion, it is easy to see that the rank of $L$ is equal to $n-1$ (since $G$ is connected) with its null space consisting of all 1's vector. Therefore, for any vector $y \in \mathbb{R}^{V}$ which is orthogonal to all 1's vector $(b \perp 1), L x=y$ has a solution. We will use $L^{\dagger}$ to denote the pseudo-inverse of $L$. Starting with the seminal work of Spielman and Teng [25], many near-linear time algorithms $[6,17]$ have been developed for solving Laplacian systems of the form $L x=b$.

\subsection{Electrical Flows and Effective Resistances}

In this section, we will go over the known connection between electrical flows and Laplacian systems [10]. As we mentioned in the algorithm overview section, we view the graph as wires of resistance $w_{e}$ and the nodes as connection points of wires. If $\varphi \in \mathbb{R}^{V}$ is a vector of potentials, then by Ohm's law, the electrical flow is given by $f=\nabla \varphi$ with the corresponding demand being $\Delta f=$ $\Delta \nabla \varphi=L \varphi$. Thus the vector of node potentials that induce a unit flow from $s$ to $t$ is given by $\varphi^{s t}=L^{\dagger}\left(\chi_{s}-\chi_{t}\right)$. Consequently, the electrical flow from $s$ to $t$ is given by $f=\nabla \varphi^{s t}=\nabla L^{\dagger}\left(\chi_{s}-\chi_{t}\right)$. For example, the flow on an edge $(u, v)$ is given by:

$$
f_{(u, v)}=\frac{1}{w_{u v}}\left[\varphi^{s t}(u)-\varphi^{s t}(v)\right]=\left(\chi_{u}-\chi_{v}\right)^{T} \nabla L^{\dagger}\left(\chi_{s}-\chi_{t}\right) .
$$

An immediate consequence of this relation is that the electrical flow is circulation-free.

The effective resistance between $s$ and $t, R(s, t)$, is defined as:

$R_{\mathrm{eff}}(s, t)=\left(\varphi^{s t}\right)^{T} L \varphi^{s t}=\left(\chi_{s}-\chi_{t}\right)^{T} L^{\dagger}\left(\chi_{s}-\chi_{t}\right)=\varphi^{s t}(s)-\varphi^{s t}(t)$.

One can easily see that $R_{\mathrm{eff}}(s, t)=\sum_{e} w_{e} f_{e}^{2}=f^{T} C^{-1} f$.

We can also view the effective resistance $R_{\mathrm{eff}}(s, t)$ and the electrical flow $f$ from $s$ to $t$ as the optimum value and solution of $\min \sum_{e} w_{e} f_{e}^{2}$ subject to $\Delta f=\chi_{s}-\chi_{t}$. This perspective lets us compare electrical flows against shortest paths and maximum flows:

- Shortest path problem corresponds to minimizing $\ell_{1}$ norm of $f$ : Since $\ell_{1}$ norm prefers sparse solutions, the shortest path(s) are quite fragile and not robust.

- Maximum flow problem corresponds to minimizing $\ell_{\infty}$ norm of $f$ : In this case, the paths found are well spread and therefore robust. However there is no more a guarantee on their lengths.

Minimizing $\ell_{2}$ norm of the flow vector might be considered a combination of these two, in that it will favor short but diverse paths.

\subsection{Schur Complements}

An important part of our preprocessing algorithm is reducing the graph size, and solving the electrical flow equation only on the smaller graph. Schur complement corresponds to Gaussian elimination applied to whole blocks at the same time. For example, if we have a symmetric block matrix $M=\left[\begin{array}{cc}A & B \\ B^{T} & C\end{array}\right]$ and $C$ is invertible, then the Schur complement of $C, M / C$ is given by $A-B C^{-1} B^{T}$. It is known that Schur complements are commutative (changing the order of complements yields the same matrix) and they are closed for Laplacian matrices (any Schur complement of a Laplacian matrix is Laplacian). If $L$ is a Laplacian matrix, and $A$ is a subset of nodes, we will use $L / A$ as a shorthand notation for the Schur complement of the principal minor corresponding to $A$ in $L$.

The usefulness of Schur complements for electrical flow computation is exemplified with the following theorem. Its proof can be found in Appendix A.1.

TheOREM 1. Given a Laplacian matrix $L \in \mathbb{R}^{V \times V}$ of a connected graph and a vector of demands $d \in \mathbb{R}^{V}$ with support $S$, the corresponding electrical flow is given by

$$
\nabla \cdot\left[\begin{array}{c}
I \\
-L_{U, U}{ }^{-1} L_{U, S}
\end{array}\right](L / U)^{\dagger} d_{S},
$$

where $U:=V \backslash S$ and $L / U$ is the Schur-complement of $U$.

\section{ALGORITHM FOR ROBUST ROUTING}

Our routing algorithm is based on finding the electrical flow from source to destination, and then decomposing it into paths. In a naive implementation of this algorithm, the running time will be dominated with the linear system solver. Even though theoretically near-linear time algorithms are known for solving Laplacian systems; they have big constants in their running times and they tend to be two orders of magnitude slower than Dijkstra.

One of our main contributions is a fast electrical flow solver algorithm that gets around this issue with some preprocessing. Our 
preprocessing step relies on partitioning the input graph into balanced components and computing the Schur complements of each component individually. We then solve the electrical flow equation on a much smaller graph, and then extend the solution to the whole graph. We want to emphasize that our algorithm is exact, in the sense that it finds the correct electrical flow solution (up to a desired accuracy, of course). Our algorithms are given in PrEProcessGrapH(Algorithm 1), Find-Electrical-Flow(Algorithm 2) and Generate-Alternates(Algorithm 3). We will first begin with an explanation of how our algorithm work, and then we will have a more detailed discussion of each step in their respective sections.

\subsection{Partitioning the Graph}

Our preprocessing step relies heavily on a good partitioning of the underlying graph $G$. We assume $G$ is partitioned into $k$ disjoint components, $\mathcal{P}=\left\{C_{1}, C_{2}, \ldots, C_{k}\right\}$, so that $V(G)=\bigcup_{i} C_{i}$. For each component $C \in \mathcal{P}$, we will use $\partial C$ and $\operatorname{int}(C)$ to refer to its boundary and interior nodes, respectively. Basically int $(C)$ is the set of nodes whose neighbors are all inside $C$, and $\partial C$ is the set of nodes incident to at least one node outside $C$ so that $\operatorname{int}(C):=\{u \in C: N(u) \subseteq C\}$ and $\partial C:=C \backslash \operatorname{int}(C)$. We will use $\partial \mathcal{P}$ and $\operatorname{int}(\mathcal{P})$ to denote the set of all boundary and interior nodes. Ideally, the partitioning should have two properties:

- Each component $C \in \mathcal{P}$ is balanced, in the sense that $|C|=$ $\Theta\left(\frac{n}{k}\right)$; and the induced subgraph $G[C]$ is connected.

- Each component cuts few edges. In particular, $|E(C, \bar{C})| \leq$ $O\left(|C|^{\gamma}\right)$ for some $\gamma \leq \frac{1}{2}$.

It is known that road networks have good partitionings [3, 9, 11, 24] with $\gamma \rightarrow \frac{1}{3}$ and one can find such partitioning efficiently. As a comparison, planar graphs only have such partitionings with $\gamma=\frac{1}{2}$, which is tight as can be seen from the grid graph.

For the simplicity of analysis, we stated that the requirements should hold for each component, however this is not really necessary. As long as these properties hold for most components, our analysis still go through. For example, in terms of expansion, as long as the average $|E(C, \bar{C})|$ and $|E(C, \bar{C})|^{2}$ are small, it is sufficient for us.

\subsection{Algorithm}

The main idea behind our solver is to find the electrical flow on the induced subgraph $G[\partial \mathcal{P}]$. Then we can treat the divergence of this flow on the boundary nodes as demands for that respective component, and solve for the electrical flow with the new demands on each component separately. Moreover, if there are no external demands in that component, the electrical flow computation can be expressed as a matrix multiplication, with a matrix of size number of interior nodes-by-number of boundary nodes, which can be precomputed beforehand.

As an intuition behind how to compute the flow on $G[\partial \mathcal{P}]$, let's consider a single component $C$. As discussed in Section 2.1, we need to first find the node potentials, $\varphi$, for finding the electrical flow. The node potentials are a solution of the linear system $L \varphi=d$, where $d$ is the demands vector $\left(d=\chi_{s}-\chi_{t}\right)$.

We will use $U$ to denote the rest of the nodes $(U=V \backslash C)$. Since there is no edge between $\operatorname{int}(C)$ and $U$, the corresponding block
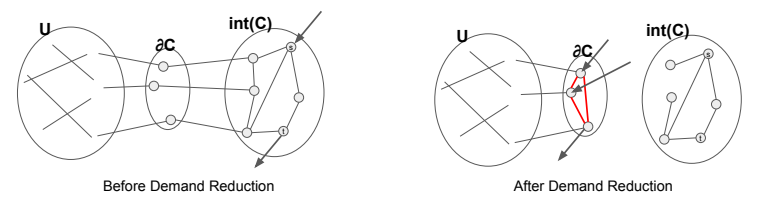

Figure 1: Illustration of demand reduction as described in Section 3.2. Taking the Schur complement of int $(C)$ adds new edges between the boundary nodes $\partial C$ while removing the edges between $\partial C$ and $\operatorname{int}(C)$. Also it transforms the demands on $\operatorname{int}(C)$ to demands on boundary, $\partial C$, which corresponds to the multiplication with the transfer matrix $-Y$ and adding the demands to the $\partial C$.

in the Laplacian matrix, $L_{\operatorname{int}(C), U}$, is 0 . Thus, by re-ordering the nodes if necessary, we can express the linear system we are trying to solve as the following:

$$
\left[\begin{array}{ccc}
L_{U, U} & L_{U, \partial C} & 0 \\
L_{\partial C, U} & L_{\partial C, \partial C} & L_{\partial C, \operatorname{int}(C)} \\
0 & L_{\operatorname{int}(C), \partial C} & L_{\operatorname{int}(C), \operatorname{int}(C)}
\end{array}\right] \cdot\left[\begin{array}{c}
\varphi_{U} \\
\varphi_{\partial C} \\
\varphi_{\operatorname{int}(C)}
\end{array}\right]=\left[\begin{array}{c}
d_{U} \\
d_{\partial C} \\
d_{\operatorname{int}(C)}
\end{array}\right] .
$$

Let $Y:=L_{\partial C, \operatorname{int}(C)} L_{\operatorname{int}(C), \operatorname{int}(C)}^{-1}$. If we multiply on the left with the invertible matrix $\left[\begin{array}{ccc}I & 0 & 0 \\ 0 & I & -Y \\ 0 & 0 & I\end{array}\right]$, the top two rows become: $\left[\begin{array}{ccc}L_{U, U} & L_{U, \partial C} & 0 \\ L_{\partial C, U} & L_{\partial C, \partial C}-L_{\partial C, \operatorname{int}(C)} Y & 0\end{array}\right] \cdot\left[\begin{array}{c}\varphi_{U} \\ \varphi_{\partial C}\end{array}\right]=\left[\begin{array}{c}d_{U} \\ d_{\partial C}-Y d_{\text {int }(C)}\end{array}\right]$.

Let $\widehat{L}$ be the top-left $2 x 2$ block of above matrix. It is easy to see that $\widehat{L}$ is the Schur-complement of $\operatorname{int}(C)$, therefore $\widehat{L}$ itself is a Laplacian matrix. Hence we can reduced the problem to finding potentials on $V^{\prime}:=U \cup \partial C$, with the new demands $d^{r}:=\left[\begin{array}{c}d_{U} \\ d_{\partial C}-Y d_{\operatorname{int}(C)}\end{array}\right]$. Intuitively multiplication with the matrix $-Y$ allows us to transfer the demands from int $(C)$ to $\partial C$. We illustrate this demand reduction in Figure 1.

Now suppose we solved the system $\widehat{L} \varphi^{r}=d^{r}$, and obtained potentials for the reduced graph. At this point, it might be tempting to use the reduced potentials as the "true potentials" of the nodes $V^{\prime}$ and use these to compute the potentials of the remaining nodes. However, this approach does not work, $\widehat{L}$ is not an invertible matrix and we can not determine $\varphi^{r}$ uniquely (the null-space contains all 1's vector, so $\varphi^{r}$ might be shifted). Instead, we can use $\varphi^{r}$ to compute the flows on edges of $G\left[V^{\prime}\right], E^{b}$, which are unaffected by shifts.

Having found the partial flow on edges of $G\left[V^{\prime}\right]$, our final task is to compute the flow on the remaining edges, $E^{i}$, which are the edges incident to the interior nodes, $\operatorname{int}(C)$. Consider any boundary node $u \in \partial C$. Because of flow conservation, the net flow on $u$ due to the edges from $E^{b}$ has to be met by the net flow on the edges of $E^{i}$ plus the initial demand $d_{u}$. This gives us a new set of demands $d^{\prime}$ on $C$, and the flow on edges $E^{i}$ should satisfy the electrical flow equation with respect to these demands (see Figure 2 for an illustration). In particular, if we let $\mathcal{H}$ be the graph $G[C]$ with edges between $\partial C$ removed, then the flow on the edges of $\mathcal{H}$ will be equal to $\nabla_{\mathcal{H}} \cdot \varphi$ where $\varphi_{C}$ is the potentials in $\mathcal{H}$ with respect to demands $d^{\prime}$. To 


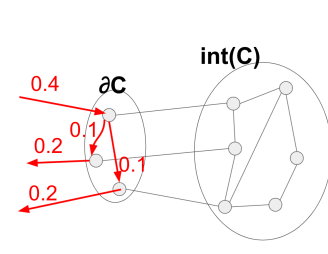

Flow on Boundary Edges

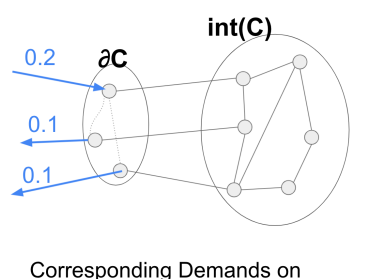

Boundary Nodes
Figure 2: Illustration of how the flow on boundary edges are transformed to demands on the boundary nodes as described in Section 3.2. The red edges on the left graph correspond to the flows on boundary edges. The graph on the right corresponds to $\mathcal{H}$. We can preserve the flow at each boundary node by adding the demands shown by the blue edges on the right.

complete our computation, we need to find a solution of the system $\mathcal{L} \varphi_{C}=d^{\prime}$ (where $\mathcal{L}$ is the Laplacian of $\mathcal{H}$ ), and then let the flow on edges of $\mathcal{H}$ be $\nabla_{\mathcal{H}} \cdot \varphi_{C}$.

The final ingredient in our algorithm is speeding-up this last step. If we examine the new demands on interior nodes, $d_{\text {int }(C)}^{\prime}$, we can see that they are equal to the original demands. However, originally there are demands only on two nodes, which means at most two components can have a non-zero demand in their interior Suppose our component, $C$, do not have any interior demand so that $d_{\operatorname{int}(C)}^{\prime}=0$. Then we can use Theorem 1 to obtain a linear operator that maps the demands on boundary to a potential vector, whose gradient gives the electrical flow on $\mathcal{H}$ :

$$
\varphi_{C}=\underbrace{\left[\begin{array}{cc}
I \\
-\mathcal{L}_{\operatorname{int}(C), \operatorname{int}(C)}{ }^{-1} \mathcal{L}_{\operatorname{int}(C), \partial C}
\end{array}\right](\mathcal{L} / \operatorname{int}(C))^{\dagger}}_{:=X} d_{\partial C} .
$$

Note that the matrix $X$, just like the matrix $Y$, can be computed and stored beforehand and re-used.

We can keep repeating this process for each component $C$, each time eliminating that component's interior nodes and updating the reduced demands, $d^{r}$. At the end, we will end up with the linear system $\widehat{L} \varphi^{r}=d^{r}$, which can be solved using any of the efficient Laplacian solvers $[6,17,25]$. Furthermore the matrices $\widehat{L}, X$ and $Y$ can be precomputed.

\subsection{Choice of Laplacian System Solver}

Provided the partition size $\left(\frac{n}{k}\right)$ is kept reasonably low; the choice of linear system solver has little affect on the running time of Preprocess-Graph (Algorithm 1). The biggest affect will be observed in Find-Electrical-Flow (Algorithm 2), where we need to solve a linear system involving $\widehat{L}$. For practical purposes, one can build a preconditioner for $\widehat{L}$ at the end of processing stage and pass it along the other parameters to GenERATE-Alternates and Find-Electrical-Flow. In our implementation, we used incomplete Cholesky factorization with thresholding to compute a preconditioner, and used preconditioned conjugate gradient (PCG) algorithm as our linear system solver.

In order to study the worst case running time of our algorithms, we will assume the linear system solver takes time $O\left(m \log ^{c} n\right)$ on
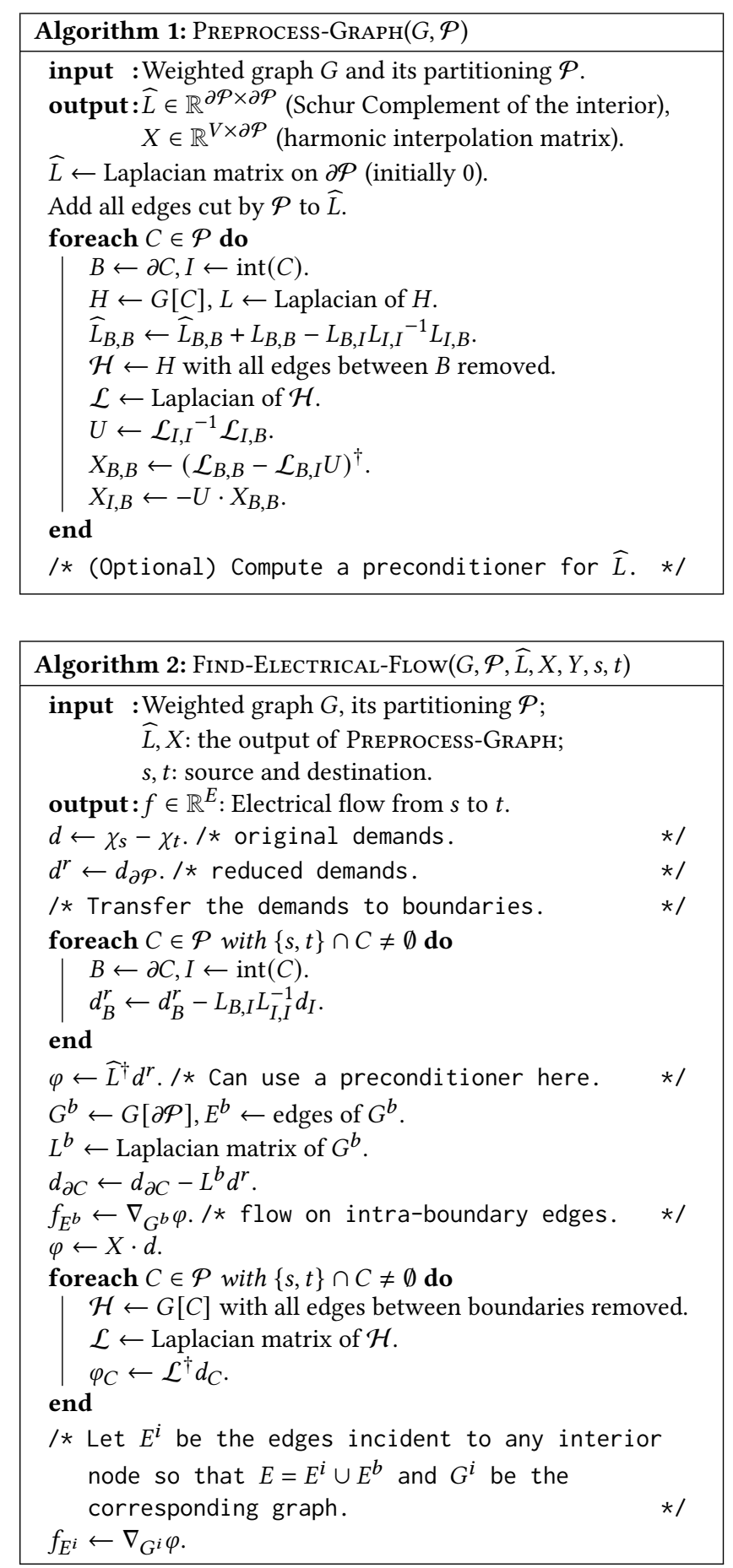

a Laplacian system with $n$ nodes and $m$ edges. As of now, the fastest solver in theory has $c=\frac{1}{2}+\epsilon$ as $\epsilon \rightarrow 0$ [6].

\subsection{Preprocessing}

The preprocessing step is given in Preprocess-GrapH (Algorithm 1). The computations for each component $C$ are independent of each other, so they can all be run in parallel. The algorithm closely follows the description in Section 3.2, and computes the matrices $\widehat{L}$ 


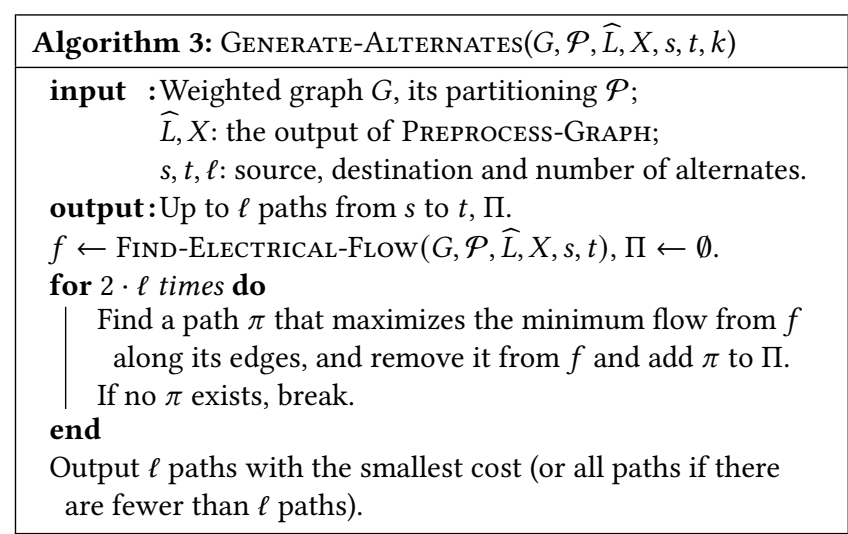

and $X$ to be used later. Given the derivations in Section 3.2, the correctness of Preprocess-Graph is immediate. In this section, we will analyze its time and space complexity.

We will first bound the number of non-zeros (nnz) in the matrices $\widehat{L}$ and $X$. Then we will analyze the space and time complexity of Preprocess-Graph. Proofs of Lemmas 1 and 2 and Theorem 2 could be found in Appendix A.1.

LEMMA 1. $\operatorname{nnz}(\widehat{L}) \leq O\left(k^{1-2 \gamma} n^{2 \gamma}\right)$.

LEMmA 2. $\mathrm{nnz}(X) \leq O\left(n^{1+\gamma} k^{-\gamma}\right)$.

TheOREM 2. Under the assumptions on the partitioning $\mathcal{P}$ from Section 3.1, PREPROCESS-GRAPH (Algorithm 1) runs in space $O\left(n^{1+\gamma} k^{-\gamma}\right)$ and time $O\left(n^{1+\gamma} k^{-\gamma} \log ^{c}(n / k)\right)$.

\subsection{Alternate Generation}

Our alternate generation algorithm is given in GenERATE-Alternates (Algorithm 3). It uses Find-ElectricAL-Flow(Algorithm 2) as a subroutine. The input of this algorithm is the $\widehat{L}, X$ and $Y$ matrices, which are computed during the preprocessing stage. The correctness of the algorithm easily follows from Section 3.2.

LEMMA 3. Under the assumptions on the partitioning $\mathcal{P}$ from Section 3.1, FIND-ELECTRICAL-FLOW (Algorithm 2) runs in time $O\left(n^{1+\gamma} k^{-\gamma}\right)$.

Proof. Solving the first linear system takes $O\left(n n z(\widehat{L}) \log ^{c}(\mathrm{nnz}(\widehat{L}))\right)$ time which is $O\left(k^{1-2 \gamma} n^{2 \gamma} \log ^{c}(n)\right)$ using Lemma 1. The next linear system solutions (in the for loop) take $O\left((n / k) \log ^{c}(n / k)\right)$ time. Finally the matrix multiplications take $O(n n z(X))$ time, which is $O\left(n^{1+\gamma} k^{-\gamma}\right)$ from Lemma 2 , dominating the running time.

REMARK 1. In FIND-ELECTRICAL-FLOW (Algorithm 2), with the exception of step $\varphi \leftarrow \widehat{L}^{\dagger} d^{r}$, all other steps can trivially be parallelized, including the step that dominates the running time that involves the matrix-vector multiplication $X \cdot d$.

We are finally ready to state the worst case running time of Generate-Alternates (Algorithm 3).

Theorem 3. Under the assumptions on the partitioning $\mathcal{P}$ from Section 3.1, GenERATE-ALterNates (Algorithm 3) runs in time $O\left(n^{1+\gamma} k^{-\gamma}+\ell n\right)$.

The running time bound in Theorem 3 immediately suggest an optimal value for the number of partitions, $k$.
Corollary 1. Given a $k$-way partitioning $\mathcal{P}$ where $k=\frac{n}{\ell^{1 / \gamma}}$ satisfying the requirements from Section 3.1, PREPROCESS-GRAPH runs in time $O\left(n \ell \log ^{c} \ell\right)$ and uses space $O(n \ell)$; while GENERATE-ALTERNATES runs in worst case time $O(n \ell)$. The running time is optimal as the output could be of size $\Theta(n \ell)$.

Proof of Theorem 3. The running time of Find-ElectricalFlow is $O\left(n^{1+\gamma} k^{-\gamma}\right)$ as shown in Lemma 3. We can implement the second step of the algorithm (finding max-min flow paths) to run in worst-case time $O(\mathrm{~m})$ per path, by observing that the flow graph is a directed acyclic graph. Hence we can use linear time dynamic programming to find each max-min flow path.

Notice that, in practice, the most costly step of our algorithm is the flow decomposition for large number of alternates. Even though linear time dynamic programming makes sense from a worst case perspective; in practice, running Dijkstra (using the semiring $(\max , \min )$ instead of $(\min ,+))$ is much faster. Although the naive implementation of this step will run in time $O(\ell n \log n)$, there are certain heuristics that could be made, which speeds up the running time considerably:

- We sparsify the flow graph by removing any edge that has too little flow on it (we used $10^{-7}$ as a threshold).

- During the Dijkstra for max-min flow path selection, if the node we consider has only a single outgoing edge, we can keep following that edge until we hit an already visited node. Since the flow on the outgoing edge can never be less than the incoming one, that means we found the max-min flow path to each of the nodes we visited along the way. In our experiments, this heuristic provided the most speed up.

\section{EXPERIMENTAL EVALUATION}

In this section, we evaluate the performance of our alternates generation algorithm on real road networks. We show that our algorithm produces alternates that are more robust and diverse than the plateau method [1] while having a comparable running time.

In the following subsections, we describe the data used to construct our experimental instances, the exact process followed to conduct our experiments, and the main conceptual conclusions we reach. Finally, we present our results in detail.

\subsection{Setup}

Graphs. All our algorithms are performed on Open Street Map [20] data. We conduct experiments on the Bay Area region (containing San Francisco and San Jose). To run experiments on this area, we clip the map using lat-long boundaries. We compute the weight of each directed edge as the ratio of that edge's distance and the maximum speed along that edge. Next, we make the graph undirected and remove parallel edges by only keeping the edge with minimum weight. The clipping leads to multiple disconnected components near the edges. To take care of that, we use the largest among them as the map for the respective city.

Statistics. Our graph has 2.73M nodes and 2.93M edges. We used the Inertial Flow algorithm [24] with balancedness parameter 0.1 to compute a partitioning of our graph with the partition sizes being between 250 and 500 . There were $9.3 \mathrm{~K}$ components in the 
partitioning. The matrices $\widehat{L}, X$ and $Y$ generated by PreprocessGrAPH (Algorithm 1) (as explained in Section 3.2) had $\mathrm{nnz}(\widehat{L})=$ $615 K, \mathrm{nnz}(X)=19.3 M$ and $\mathrm{nnz}(Y)=18.7 M$ non-zero entries, respectively.

Source-Destination Pairs. We randomly sampled 200 source-destination pairs from $10 \mathrm{~km}$ up to a distance of $100 \mathrm{~km}$ (or miles) while making sure that the distribution of distances was uniform (i.e., 20 pairs have a distance between 0 and $10 \mathrm{~km} ; 100$ pairs have a distance between 10 and $20 \mathrm{~km}$ and so on). For each pair, we generated 20 and 100 alternate paths.

Solving Linear Systems. We used Preconditioned Conjugate Gradient algorithm and an incomplete Cholesky factorization preconditioner with thresholding [22], where we set the drop threshold to $10^{-7}$. Implementation Details. All our experiments are conducted on a standard MacBook Pro 2019, running on macOs Big Sur 11.3.1, with 16GB Ram and 8-Core $2.3 \mathrm{GHz}$ Intel Core i9 processor. All our algorithms are implemented in $\mathrm{C}++$ with Apple clang compiler version 12 . We used 4-way heap to implement Dijkstra.

\subsection{Plateau and Penalty Methods}

In this section, we will provide a detailed explanation of our implementations for plateau and penalty methods.

Penalty Method. In the penalty method [4], we repeatedly choose a shortest path, and increase the weight of all edges along and incident to that path by a factor of $\beta=1.20$. We keep iterating this procedure until we obtain $K$ distinct paths or $2 \cdot K$ iterations reached. We used pruning [21] to speed up the algorithm, and restrict the search space to only nodes $u$ such that $d(s, u)+d(u, t) \leq \Delta_{0} d(s, t)$ for $\Delta_{0}=2$. However pruning has little to no effect as the distance increase. If the maximum number of iterations are reached, yet not enough alternates are found (or they are not diverse enough), then we multiply $\Delta_{0}$ by a factor of 1.5 , and re-do the computation ${ }^{1}$.

This method is known to generate high quality alternates. However the output and performance are very sensitive to the penalty function, $\beta$. In order to obtain good results, the penalty parameter needs to be made smaller; however smaller values of $\beta$ make the algorithm prohibitively slow. Even with reasonably high penalty values, this method is quite slow: After each update, it is necessary to run a full Dijkstra. Since the graph keeps changing between iterations, it is not possible to speed up this step using any of the known preprocessing techniques.

Plateau Method. In the plateau method, we construct a forward shortest path tree from the source and a backward shortest path tree from the destination. All edges present in both of the trees are called plateau edges. Note that each plateau edge defines a unique path from source to sink in the union of these two trees. Next all plateau edges are sorted with respect to the length of the corresponding source-destination path. Finally we go through each such path, and add it to the set of alternates as long as its minimum Jaccard distance with any of the found paths is greater than some threshold $\sigma$. If the method fails to produce the desired number of alternates, we decrease the threshold and retry again. For our experiments, the thresholds we used are $\{0.3,0.2,0.1\}$. To speed up

\footnotetext{
${ }^{1}$ However, this step never happened in our experiments and for our initial choice of $\Delta_{0}$, the penalty method always found the desired number of diverse paths within the allowed number of iterations.
}

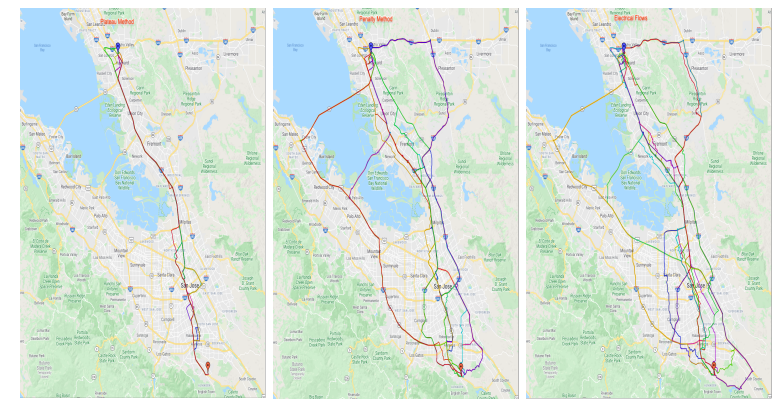

Figure 3: 20 alternates generated by each method for the same source-destination point. Plateau method failed to produce any alternate that avoids a major highway (Interstate 880 ), whereas both penalty and electrical flows found alternates utilizing all major roads.

the algorithm, after we consider an edge, we removed the preceding and proceeding 50 edges in the forward and backward trees from further consideration. Plateau method gives paths with minimum stretch possible. However it also has also some drawbacks:

- The alternates produced by plateau method tend to be very similar to each other. On the other hand, it can not find a reasonable number of alternates if the threshold is set high.

- If there are not enough alternates for the given similarity threshold, plateau method will run very slow as nearly all the plateau edges need to be expanded.

- When the source and destination both are very close to a shortcut road (such as a highway), all the alternates will be using that shortcut road (see Figure 3 for an example).

Figure 3 shows 20 alternates generated by each algorithm for two sets of source-destination pairs. As it can be seen from the figures, all the alternates generated by the plateau method used the same highway, whereas both penalty and electrical flows generated a diverse set of alternates.

\subsection{Running Times}

Our first results, in Figure 4, are running time comparisons between our electrical flow algorithm (EF), plateau (PLA) and penalty (PEN) methods. For each source-destination pair, we recorded the ratio of running times for PLA and PEN against EF for generating 20 and 100 alternates. Since the algorithms exhibit different run-time behaviour with respect to the distances, we found it most informative to present the ratio of running times averaged over $10 \mathrm{~km}$ buckets. For 20 alternates, the running times of PLA and EF were comparable across all distances; however for 100 alternates, the gap between PLA and EF widens with EF being four times faster than PLA at $>100 \mathrm{~km}$. This is mostly due to the fact that PLA needs to examine more plateau edges, which involves expanding the corresponding paths and running the similarity checks, as the number of requested alternates increase. On the other hand, the running time of EF is quite consistent and changes very little with respect to distances. Finally PEN method has the slowest running time: Even for 20 alternates, for large distances, it runs nearly 10 times slower than EF.

For our second experiment, we analyzed the affect of number of paths and distance on the average running time of EF algorithm 
per path. Our results can be found in Figure 5. We observed that, for 20 alternates, the running time does not change with respect to distance confirming our observation from Figure 4. Suprisingly, for 100 alternates, the average running time is faster than 20 alternates. This means the dominating factor in the running time of our EF algorithm is Find-ELECTRICAL-Flow(Algorithm 2), whose running time is independent of the number of alternates, as opposed to the flow decomposition step of GenERATE-Alternates(Algorithm 3).

\subsection{Stretch and Diversity}

Given a sequence of alternate paths $\left(\pi_{1}, \pi_{2}, \ldots, \pi_{k}\right)$, we investigate the quality of each alternate through three different quantities.

Stretch. One natural constraint for any alternate generation algorithm is that the produced alternates should not be much worse than the shortest path. We quantify this by measuring the stretch of each path, which is the ratio of path's cost to the shortest path cost. Stretches are given in Figure 6 for 20 and 100 alternates. Rather surprisingly, the stretches are somewhat uniform across all distances. As expected, PLA has the smallest stretch uniformly across all distances and for any number of alternates. For 20 alternates, PEN's stretch, which is $25 \%$ on average, is consistently better than EF, which is $37 \%$ on average. However we see that this trend is reversed for 100 alternates: EF is consistently better, with an average stretch of $70 \%$ whereas PLA has an average stretch of $95 \%$.

Diversity. Another desirable aspect from alternate paths is that each path should be sufficiently different from all the preceding ones. For this we use Jaccard distance, $J(A, B):=\frac{|A \Delta B|}{|A \cup B|}$, where $A \triangle B$ is the symmetric set difference. For each path $\pi_{i}$, we record the minimum Jaccard distance to the preceding paths, $\max _{j<i} J\left(\pi_{i}, \pi_{j}\right)$, where we view each path $\pi_{i}$ as a set over edges. The diversity results for 20 and 100 alternates are given in Figure 7. As expected, the diversity of PLA is never better than the diversity threshold $\sigma=0.3$ and it is much worse than both EF and PEN. For both 20 and 100 alternates, the paths produced by EF are usually more diverse than PEN's paths. The diversity of EF is aligned with our expectations as the flow decomposition method disallows sharing of edges between paths.

\subsection{Robustness Under Random Edge Deletions}

One noticeable shortcoming of the Jaccard similarity is its pairwise nature - even though any pair of paths might have very low similarity, it is possible that they are all different combinations of a small number of segments. Therefore, such a diversity measures will not be indicative of the robustness of produced alternates to failures. Perhaps the simplest failure model is random edge deletion. What fraction of the edges can we delete independently at random and still find a path from source to destination in the directed alternates graph? We want to compute the maximum fraction of edges that can be randomly deleted before the probability of $t$ being unreachable from $s$ is at least $\frac{1}{2}$.

We are not aware of an efficient algorithm that computes this "connectivity threshold" for general graphs. For our experiments, we used the following approximation algorithm for $\rho$ (averaged over 30 runs):

(1) Choose a random ordering of the edges, $o: E \rightarrow \mathbb{Z}$.
(2) Find the path that maximizes the minimum $o_{e}$ along its edges in the directed alternates graph. Let $o^{*}$ be this value and output $\frac{o^{*}}{|E|}$.

The relative robustness probabilities with respect to PLA could be found in Figure 8 . The alternatives found by both PEN and EF are resistant to nearly twice more edge failures than PLA. As opposed to diversity, here we see that the alternates found by PEN are $10 \%$ to $15 \%$ more robust than EF's alternates.

\subsection{Robustness in Terms of Effective Resistance}

Effective resistance itself can also be used as a robustness measure. It is a complex function of the network that takes into account different routes from $s$ to $t$, their stretches and overlaps. For example, in a graph where there is a single path from $s$ to $t$ of length $k$, the $s-t$ effective resistance will be $k$, whereas if there are $k$ parallel paths of length $10 k$, the effective resistance will be 10 . So a lower effective resistance indicates a more robust alternates graph. Our results for 20 and 100 alternate paths can be found in Figure 9. The results are given as ratio against PLA, whose alternates always have the highest effective resistance. Both PEN and EF consistently finds alternates whose effective resistances are $60 \%$ or lower than that of the PLA. Like the robustness case, PEN achieves $20 \%$ better effective resistances than EF.

\section{CONCLUSION}

In this work, we introduced a new algorithm for computing alternate routes in road networks using electrical flows. We showed how to find such electrical flows efficiently with some preprocessing, and proved that our algorithm has optimal worst case running time. We also implemented our algorithm in a prototype and compared it against two popular alternate generation algorithms: plateau and penalty methods. We showed that our algorithm not only outperforms these two methods in terms of running time, but it displays stable running times irrespective of the number of alternates or distances; two parameters which highly affect the running time of other methods. Then we evaluated the quality of alternates generated by our method against the other ones, and showed that our method finds diverse and robust set of alternates.

Directions for future research include generalization to directed graphs, incorporating fast updates to the preprocessing algorithm and better flow decompositions strategies to decrease the stretch.

\section{REFERENCES}

[1] 2009. Camvit: Choice routing. http://www.camvit.com.

[2] Ittai Abraham, Daniel Delling, Andrew V. Goldberg, and Renato F. Werneck. 2013. Alternative routes in road networks. ACM 7. Exp. Algorithmics 18 (2013)

[3] Gaurav Aggarwal, Sreenivas Gollapudi, Raghavender R, and Ali Kemal Sinop. 2021. Sketch-based Algorithms for Approximate Shortest Paths in Road Networks. In WWW'21: The Web Conference 2021, Ljubljana, Slovenia, April 19-23, 2021. $\mathrm{ACM} / \mathrm{IW} 3 \mathrm{C} 2$.

[4] Roland Bader, Jonathan Dees, Robert Geisberger, and Peter Sanders. 2011. Alternative Route Graphs in Road Networks. In Theory and Practice of Algorithms in (Computer) Systems, Alberto Marchetti-Spaccamela and Michael Segal (Eds.). Springer Berlin Heidelberg, Berlin, Heidelberg, 21-32.

[5] M Ben-Akiva, M J Bergman, Andrew J Daly, and Rohit Ramaswamy. 1984. Modeling inter-urban route choice behaviour. In International Symposium on Transportation and Traffic Theory. VNU Press, 299-330.

[6] Michael B. Cohen, Rasmus Kyng, Gary L. Miller, Jakub W. Pachocki, Richard Peng, Anup B. Rao, and Shen Chen Xu. 2014. Solving SDD Linear Systems in Nearly 

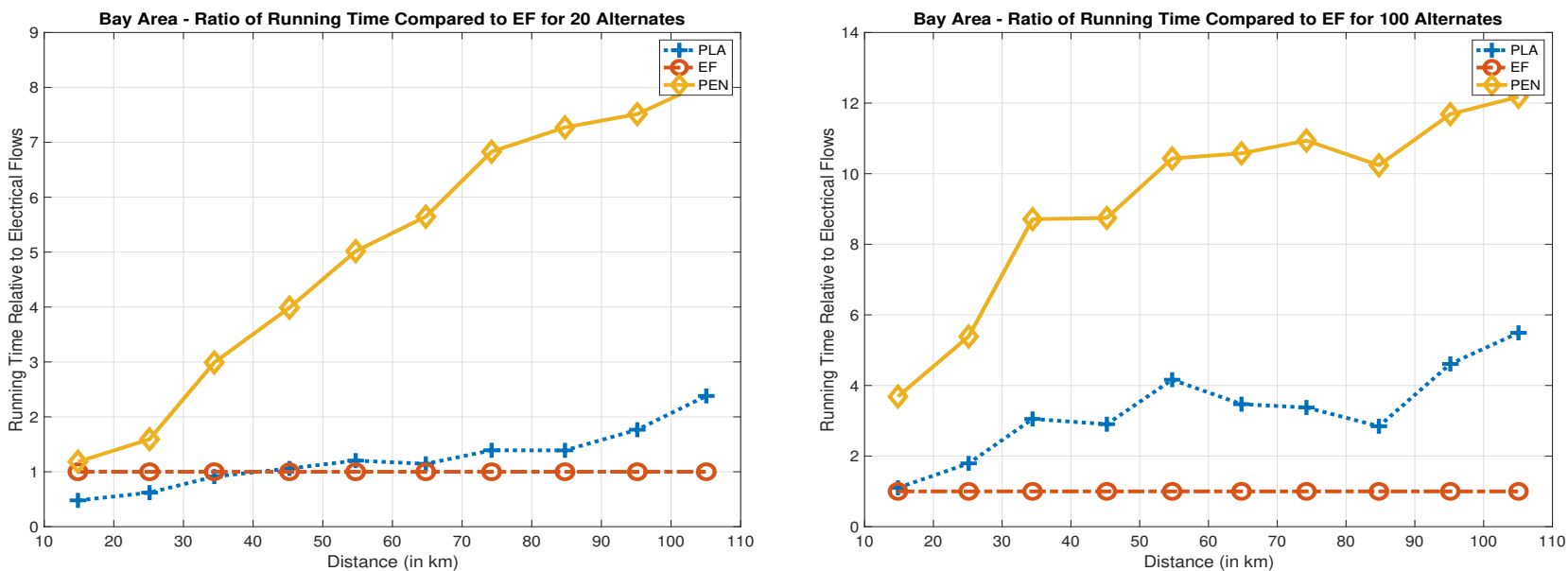

Figure 4: Running times of Plateau and Penalty as a ratio of the running time of EF algorithm for the Bay Area (Section 4.3). The query time of both PLA and PEN algorithms increases linearly relative to the EF algorithm as the distance and number of alternates increase.

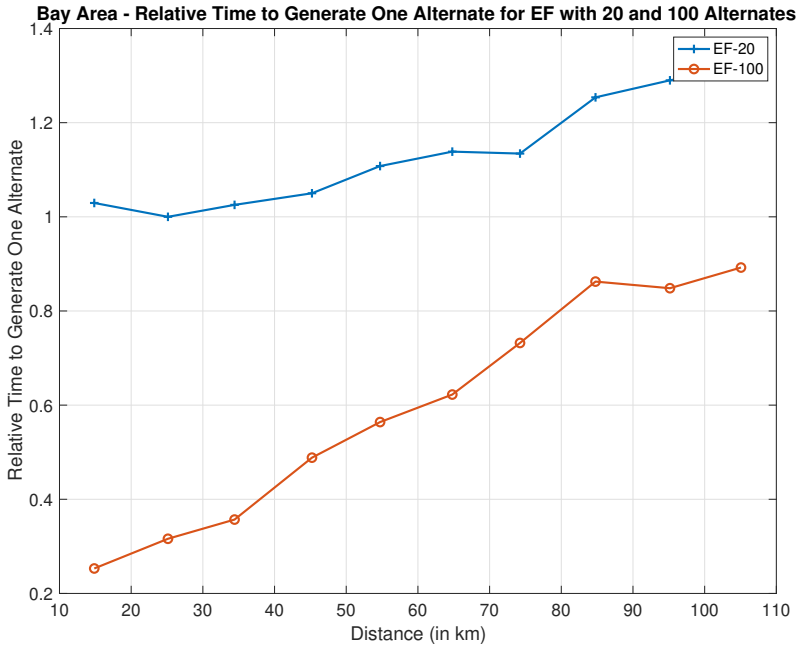

Figure 5: Change in the running time of EF algorithm with respect to different number of alternates and distances per path (Section 4.3). The average time per path decreases as the number of alternates increase.

$m \log ^{1 / 2} n$ Time. In Proceedings of the Forty-Sixth Annual ACM Symposium on Theory of Computing (STOC '14). Association for Computing Machinery, 343-352.

[7] Tomas de la Barra and B Perezand J Anez. 1993. Multidimensional path search and assignment. In PTRC Summer Annual Meeting (SAM).

[8] Daniel Delling, Andrew V. Goldberg, Thomas Pajor, and Renato F. Werneck. 2017. Customizable Route Planning in Road Networks. Transp. Sci. 51, 2 (2017), 566-591.

[9] Daniel Delling, Andrew V. Goldberg, Ilya P. Razenshteyn, and Renato Fonseca F Werneck. 2011. Graph Partitioning with Natural Cuts. In 25th IEEE International Symposium on Parallel and Distributed Processing, IPDPS 2011, Anchorage, Alaska, USA, 16-20 May, 2011 - Conference Proceedings. IEEE, 1135-1146.

[10] Peter G. Doyle and J. Laurie Snell. 1984. Random walks and electric networks. Mathematical Assn of Amer.

[11] David Eppstein and Michael T. Goodrich. 2008. Studying (non-planar) road networks through an algorithmic lens. In 16th ACM SIGSPATIAL International Symposium on Advances in Geographic Information Systems, ACM-GIS 2008, November 5-7, 2008, Irvine, California, USA, Proceedings, Walid G. Aref, Mohamed F. Mokbel, and Markus Schneider (Eds.). ACM, 16.

[12] Christos Faloutsos, Kevin S. McCurley, and Andrew Tomkins. 2004. Fast discovery of connection subgraphs. In Proceedings of the Tenth ACM SIGKDD International

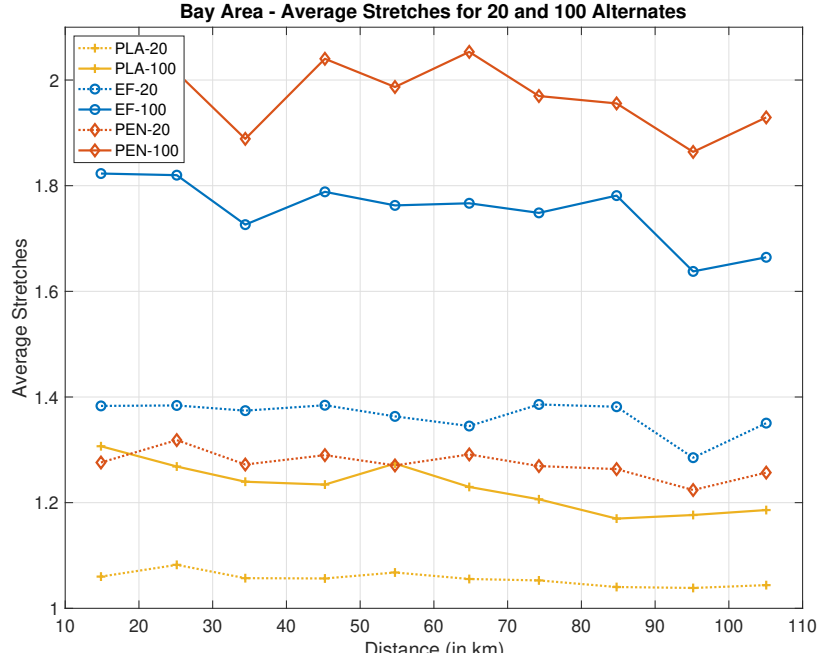

Figure 6: Average stretch of the path lengths for all algorithms for Bay Area (Section 4.4). Interestingly, the average stretch does not change much as the path length varies. As the number of alternates increase, the EF algorithm outperforms both PEN and PLA methods.

Conference on Knowledge Discovery and Data Mining, Seattle, Washington, USA, August 22-25, 2004, Won Kim, Ron Kohavi, Johannes Gehrke, and William DuMouchel (Eds.). ACM, 118-127.

[13] Robert Geisberger, Peter Sanders, Dominik Schultes, and Christian Vetter. 2012. Exact Routing in Large Road Networks Using Contraction Hierarchies. Transportation Science 46, 3 (Aug. 2012), 388-404

[14] Prahladh Harsha, Thomas P. Hayes, Hariharan Narayanan, Harald Räcke, and Jaikumar Radhakrishnan. 2008. Minimizing average latency in oblivious routing. In Proceedings of the Nineteenth Annual ACM-SIAM Symposium on Discrete Algorithms, SODA 2008, San Francisco, California, USA, January 20-22, 2008, Shang-Hua Teng (Ed.). SIAM, 200-207.

[15] Jonathan A. Kelner and Petar Maymounkov. 2011. Electric routing and concurrent flow cutting. Theor. Comput. Sci. 412, 32 (2011), 4123-4135.

[16] Moritz Kobitzsch. 2015. Alternative Route Techniques and their Applications to the Stochastics on-time Arrival Problem. Ph.D. Dissertation. Karlsruhe Institute of Technology.

[17] Rasmus Kyng and Sushant Sachdeva. 2016. Approximate Gaussian Elimination for Laplacians - Fast, Sparse, and Simple. In IEEE 57th Annual Symposium on 


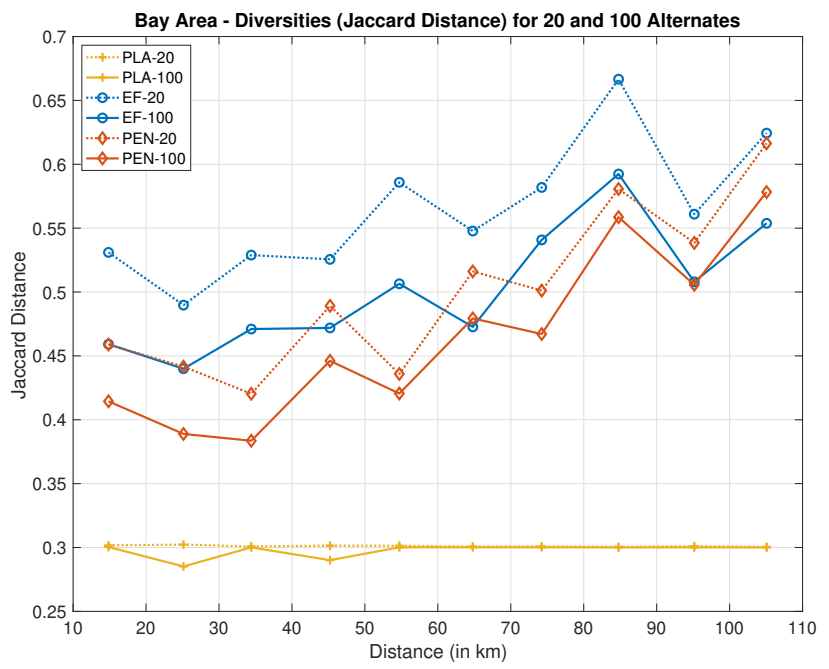

Figure 7: Diversity of the generated alternates for the Bay Area (Section 4.4). As expected, the diversity increases as the path length increases. The EF algorihm produces more diverse paths compared to the other two algorithms.

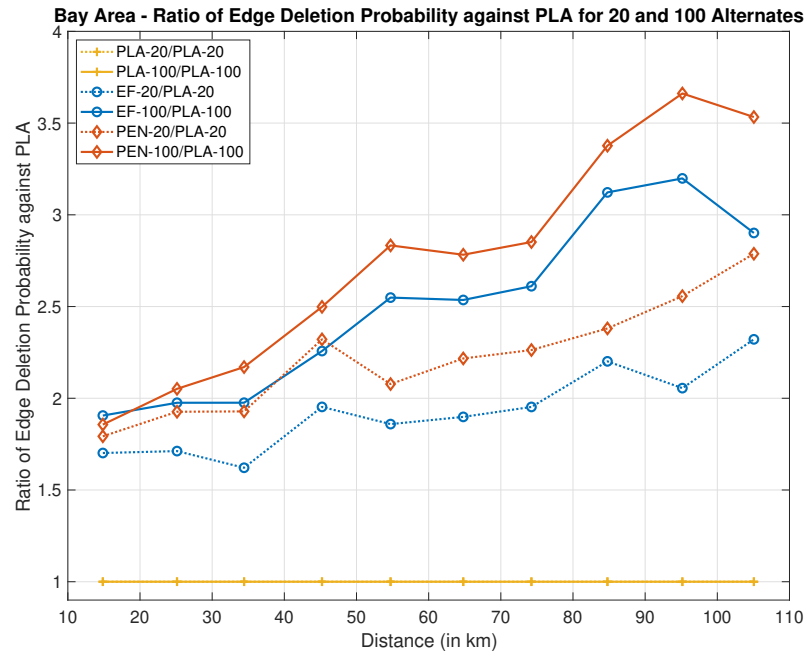

Figure 8: Relative robustness measure for Bay Area. The PEN and EF methods were compared to the PLA method which exhibited the smallest edge deletion probability at which the end points become disconnected. As the path length increases, both EF and PEN algorithms find a robust set of diverse paths.

Foundations of Computer Science, FOCS 2016, 9-11 October 2016, Hyatt Regency, New Brunswick, New fersey, USA, Irit Dinur (Ed.). IEEE Computer Society, 573-582.

[18] Gregory Lawler and Hariharan Narayanan. 2009. Mixing times and lp bounds for oblivious routing. In Proceedings of the Meeting on Analytic Algorithmics and Combinatorics. Society for Industrial and Applied Mathematics, 66-74.

[19] Dennis Luxen and Dennis Schieferdecker. 2012. Candidate Sets for Alternative Routes in Road Networks. In Experimental Algorithms - 11th International Symposium, SEA 2012, Bordeaux, France, fune 7-9, 2012. Proceedings (Lecture Notes in Computer Science), Ralf Klasing (Ed.), Vol. 7276. Springer, 260-270.

[20] OpenStreetMap contributors. 2017. Planet dump retrieved from https://planet.osm.org . https://www.openstreetmap.org.
Bay Area - Ratio of Effective Resistances of Alternate Graphs Against PLA for 20 and 100 Alternates

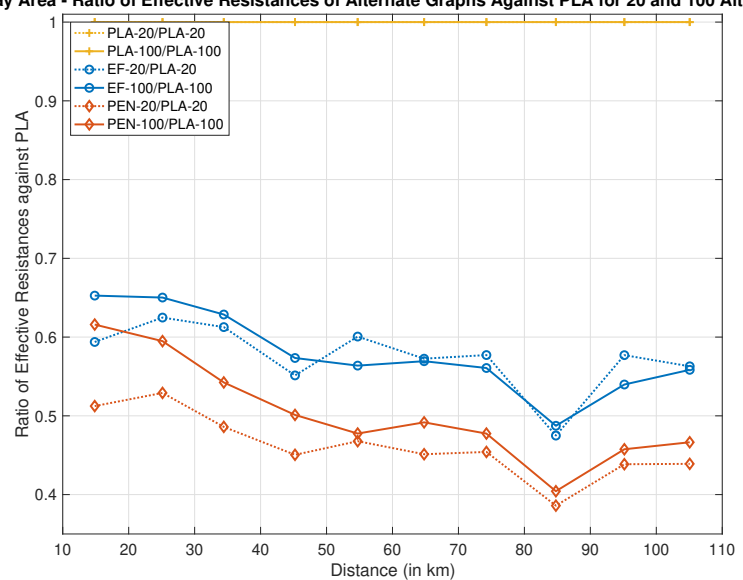

Figure 9: Effective Resistances of the induced sub-graph by the paths in the alternate set for each algorithm. A subgraph is more robust if it's effective resistance is lower. Even as the PEN method produces a slightly more robust set of paths, the EF method does very well compared the PLA method and exhibits a comparable robustness with the PEN method.

[21] Andreas Paraskevopoulos and Christos D. Zaroliagis. 2013. Improved Alternative Route Planning. In 13th Workshop on Algorithmic Approaches for Transportation Modelling, Optimization, and Systems, ATMOS 2013, September 5, 2013, Sophia Antipolis, France (OASICS), Daniele Frigioni and Sebastian Stiller (Eds.), Vol. 33. Schloss Dagstuhl - Leibniz-Zentrum für Informatik, 108-122.

[22] Yousef Saad. 1996. Iterative Methods for Sparse Linear Systems. PWS Publishing Company.

[23] Aaron Schild, Satish Rao, and Nikhil Srivastava. 2018. Localization of Electrical Flows. In Proceedings of the Twenty-Ninth Annual ACM-SIAM Symposium on Discrete Algorithms, SODA 2018, New Orleans, LA, USA, January 7-10, 2018, Artur Czumaj (Ed.). SIAM, 1577-1584.

[24] Aaron Schild and Christian Sommer. 2015. On Balanced Separators in Road Networks. In 14th International Symposium on Experimental Algorithms (SEA). 286-297.

[25] Daniel A. Spielman and Shang-Hua Teng. 2004. Nearly-linear time algorithms for graph partitioning, graph sparsification, and solving linear systems. In Proceedings of the 36th Annual ACM Symposium on Theory of Computing, Chicago, IL, USA, fune 13-16, 2004, László Babai (Ed.). ACM, 81-90.

[26] Gilbert Strang. 1986. Introduction to Applied Mathematics. Wellesley-Cambridge Press.

[27] Jin Y. Yen. 1971. Finding the K Shortest Loopless Paths in a Network. Management Science 17, 11 (1971), 712-716. 


\section{A APPENDIX}

\section{A.1 Proofs from Section 2}

Theorem 4 (Restatement of Theorem 1). Given a Laplacian matrix $L \in \mathbb{R}^{V \times V}$ of a connected graph and a vector of demands $d \in \mathbb{R}^{V}$ with support $S$, the corresponding electrical flow is given by

$$
\nabla \cdot\left[\begin{array}{c}
I \\
-L_{U, U}{ }^{-1} L_{U, S}
\end{array}\right](L / U)^{\dagger} d_{S}
$$

where $U:=V \backslash S$ and $L / U$ is the Schur-complement of $U$.

Proof. Consider the electric potentials for demands $d, \varphi$, which are a solution of the following system:

$$
\left[\begin{array}{cc}
L_{S, S} & L_{S, U} \\
L_{U, S} & L_{U, U}
\end{array}\right]\left[\begin{array}{c}
\varphi_{S} \\
\varphi_{U}
\end{array}\right]=\left[\begin{array}{c}
d_{S} \\
0
\end{array}\right]
$$

If we perform Gaussian elimination on the lower-right block (which means multiplying both sides with $\left[\begin{array}{cc}I & -L_{S, U} L_{U, U}^{-1} \\ 0 & I\end{array}\right]$ from left), we obtain:

$$
\left[\begin{array}{cc}
L / U & 0 \\
L_{U, S} & L_{U, U}
\end{array}\right]\left[\begin{array}{c}
\varphi_{S} \\
\varphi_{U}
\end{array}\right]=\left[\begin{array}{c}
d_{S} \\
0
\end{array}\right]
$$

From this, we can immediately see that $(L / U) \varphi_{S}=d_{S}$ and $\varphi_{U}=$ $-L_{U, U}{ }^{-1} L_{U, S} \varphi_{S}$. Since the original graph was connected, $L / U$ 's null-space only consist of constant vectors. Therefore we can express $\varphi_{S}$ as $\varphi_{S}=(L / U)^{\dagger} d_{S}+\alpha \mathbf{1}_{S}$ for some $\alpha$. Now we will show that the choice of $\alpha$ does not matter. Since $\mathbf{1}$ is in the null-space of $L$, we have:

$$
L_{U, S} \mathbf{1}_{S}=-L_{U, U} \mathbf{1}_{U}
$$

Given this identity, we can express $\varphi_{U}$ as:

$$
\begin{aligned}
\varphi_{U} & =-L_{U, U}{ }^{-1} L_{U, S}\left[(L / U)^{\dagger} d_{S}+\alpha 1_{S}\right] \\
& =-L_{U, U}{ }^{-1} L_{U, S}(L / U)^{\dagger} d_{S}-\alpha L_{U, U}{ }^{-1} L_{U, S} 1_{S} \\
& =-L_{U, U}{ }^{-1} L_{U, S}(L / U)^{\dagger} d_{S}+\alpha L_{U, U}{ }^{-1} L_{U, U} 1_{U} \quad \text { (using eq. (1)) } \\
& =-L_{U, U}{ }^{-1} L_{U, S}(L / U)^{\dagger} d_{S}+\alpha 1_{S} .
\end{aligned}
$$

Therefore $\varphi=\left[\begin{array}{c}I \\ -L_{U, U}{ }^{-1} L_{U, S}\end{array}\right](L / U)^{\dagger} d_{S}+\alpha \mathbf{1}$. Finally we note that $\nabla 1=0$, which means the choice of $\alpha$ does not affect our flow.

\section{A.2 Proofs from Section 2}

Lemma 4 (Restatement of Lemma 1). nnz $(\widehat{L}) \leq O\left(k^{1-2 \gamma} n^{2 \gamma}\right)$.

Proof. $\widehat{L}$ contains all the cut edges by $\mathcal{P}$ and a full matrix on the boundary of each component. Using the expansion bound of components and the fact that the boundary size of any component can be at most the number of edges cut by that component:

$\mathrm{nnz}(\widehat{L}) \leq M+\sum_{i}\left|\partial C_{i}\right|^{2}=O\left(\sum_{i}\left|C_{i}\right|^{\gamma}+\sum_{i}\left|C_{i}\right|^{2 \gamma}\right)=O\left(\sum_{i}\left|C_{i}\right|^{2 \gamma}\right)$.

Since $x^{2 \gamma}$ is a concave function for $\gamma \leq \frac{1}{2}$ and $x \geq 0$, the last expression is upper bounded by $O\left(k\left(\frac{n}{k}\right)^{2 \gamma}\right)$ which simplifies to $O\left(k^{1-2 \gamma} n^{2 \gamma}\right)$.

Lemma 5 (Restatement of Lemma 2). nnz $(X) \leq O\left(n^{1+\gamma} k^{-\gamma}\right)$.
Proof. For each component $C, X$ has a minor of size $|C| \times|\partial C|$. Therefore:

$$
\begin{aligned}
\mathrm{nnz}(X) & \leq \sum_{i}\left|C_{i}\right|\left|\partial C_{i}\right| \leq O\left(\frac{n}{k} \sum_{i}\left|\partial C_{i}\right|\right)=O\left(\frac{n}{k} \sum_{i}\left|C_{i}\right|^{\gamma}\right) \\
& =O\left(\sum_{i}\left|C_{i}\right|^{1+\gamma}\right) \leq O\left(n^{1+\gamma} k^{-\gamma}\right),
\end{aligned}
$$

where we used the fact that $\left|C_{i}\right|=\Theta(n / k)$ and $x^{\gamma}$ is concave.

Theorem 5 (Restatement of Theorem 2). Under the assumptions on the partitioning $\mathcal{P}$ from Section 3.1, PREPROCESS-GRAPH (Algorithm 1) runs in space $O\left(n^{1+\gamma} k^{-\gamma}\right)$ and time $O\left(n^{1+\gamma} k^{-\gamma} \log ^{c}(n / k)\right)$.

Proof. The space complexity follows from Lemmas 1 and 2 and the fact that $n^{1+\gamma} k^{-\gamma} \geq k^{1-2 \gamma} n^{2 \gamma}$ since $k \leq n$.

In terms of running time, in each iteration of the for loop, the most time consuming steps are the computations of $L_{I, I}^{-1} L_{I, B}, \mathcal{L}_{I, I}^{-1} \mathcal{L}_{I, B}$ and $\left(\mathcal{L}_{B, B}-\mathcal{L}_{B, I} U\right)^{\dagger}$. Since the graphs are of bounded degree, the running time of the first two operations are $O\left(|B||I| \log ^{c}(|I|)\right)$ and the last inversion takes time $O\left(|B|^{2} \log ^{c}(|B|)\right)$. So the running time is (using an argument very similar to Lemma 2):

$$
O\left(\sum_{i}\left|\partial C_{i}\right|\left|C_{i}\right| \log ^{c}\left(\left|C_{i}\right|\right)\right) \leq O\left(k^{-\gamma} n^{1+\gamma} \log ^{c}(n / k)\right) \text {. }
$$

